
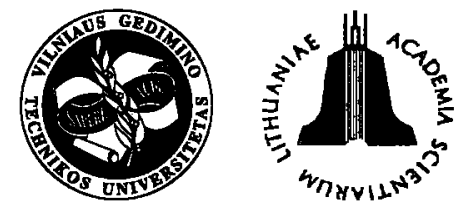

TRANSPORT

http:/www.vtu.lt/english/editions

TRANSPORT - 2002, Vol XVII, No $1,8-14$

\title{
PECULIARITIES OF TRACTION FORCES IN WHEEL/RAIL CONTACT AREA
}

\author{
Gintautas Bureika ${ }^{1}$, Šrūnas Mikaliūnas² \\ Department of Railway Transport, Vilnius Gediminas Technical University (VGTU), J. Basanavičiaus g. 28, \\ LT-2009 Vilnius, Lithuania, Tel. (22) 233-844
}

Received 200111 14; accepted 20011219

\begin{abstract}
Tractive force and train movement stability dependence on division of forces involved in wheel/rail contact area are the main subjects of this article. The influence of wheel/rail contact properties in the vehicle dynamics and adhesion fields is investigated. Current tendency all over the world is to reduce the conicity of wheels with the aim of increasing the speed of trains. Wave-length of moving wheel-set in track for worn wheel-set tyre is twice less than for new wheel running profile.

Keywords: railway, locomotive, traction force, wheel and rail contact, slip velocity, wheel profile, adhesion.
\end{abstract}

\section{Introduction}

Traction force and train movement stability dependence on division of forces involved in wheel/rail contact area are the main subjects of this article.

There is a general trend to increase train speed of railway transportation all over the world. The fast development of modern railway vehicles and increasing travelling speeds are associated with phenomena of wheel running profile and corrugation of rails $[1,2]$. There are at least four dynamic problems with the existing track structures caused by raising train speed. Firstly, increased speed brings about impacts and vibrations on turnout structures. Because of the existence of rail discontinuity in fixed frogs, impacts between wheels and frogs are becoming more severe when a train passes through a fixed frog at higher speed which may shorten the life of tumout. Secondly, much attention needs to be paid to welded rail joints on the speed-raised lines because the short-wavelength irregularities on the welded rail joints may lead to intense wheel-rail dynamic effects at higher speeds. The third problem lies in the bridge-subgrade transition sections. When a train passes through these sections at high speed, the dynamic wheel loads will fluctuate and the vehicles will vibrate strongly, influencing the riding comfort. This is because the track stiffness and deformation are different with the different supporting conditions on the bridge and on the subgrade soil. In addition, a prominent problem is the effect on track of wheel flats [3].

The running of a steel wheel on a steel rail is still the original feature of all forms of guided rail systems. The

${ }^{1}$ E-mail: gintautas.bureika@ti.vtu.lt

2E-mail:smikal@ti.vtu.lt, smikal@takas.lt respective shapes of these two components have been adapted for the dual function of support and guidance. Materials are selected so as to minimise wear and risk of breakage which obviously could have catastrophic consequences. The characteristics likely to influence the wheel/rail contact properties significantly in the areas of vebicle dynamics and adhesion are investigated here.

\section{Wheel/rail contact mechanics}

\subsection{Statistical study of wheel/rail contact reminder} of results of the Hertz theory

The Hertz theory still remains valid and is intended to calculate the dimensions and shape of the contact area between two elastic bodies that are pressed against each other and to calculate the elementary pressures in this contact area. The increase in the contact force, as compared with the static force (vertical load $F_{z}$ ), is of the order of $100 \%$ at $70 \mathrm{~km} / \mathrm{h}$ and $200 \%$ at $150 \mathrm{~km} / \mathrm{h}$ [4].

Hertz demonstrated that the contact area is elliptical and that the intensity of the unit pressures is represented by an ellipsoid bounded by the contact ellipse [5]:

$$
\sigma(x, y)=\frac{3 F_{z}}{2 \pi a b} \sqrt{1-\left(\frac{x}{a}\right)^{2}-\left(\frac{y}{b}\right)^{2}} ;
$$

where $a, b$-semi axes of the Hertz contact ellipse, $\mathrm{mm}$; $F_{z}$-vertical load, $\mathrm{kN}$.

The semi-axes $a$ and $b$ of the ellipse are determined by:

- the radii of curvature of the bodies in contact;

- the modulus of elasticity $\mathrm{E}$ of the bodies in contact; Regardless of the grade used, the modulus of elasticity remains approximately $210000 \mathrm{MPa}$ and the Pois- 
son coefficient remains approximately 0.25 ;

- the tracking force of the bodies in contact.

\subsection{Dynamic analysis}

The purpose of dynamic analysis is to calculate the forces involved in the contact area. The two bodies in contact are moved in relation to each other with a running and pivoting movement:

- running only: the quotient of the tangential force $F_{t r}$ and the normal force $F_{z}$ is a function of reduced slip $v$, i.e. the ratio of the slip velocity $v_{\text {slip }}$ to the forward velocity $v$ :

$$
v=\frac{v_{\text {slip }}}{v} \text {. }
$$

In the range of low slip (several \%o) $F_{t} / F_{z}$ can be considered as being proportional to $v$. If the slip increases, a saturation effect occurs.

The maximum value of adhesion coefficient $\psi$ depends on the physical state of the body that is in contact (dry, wet, lubricated, etc.), the tracking pressure, forward velocity $v$, etc. (Fig 1 ).

- pivoting only: the quotient of the moment of torsion

$M_{o z}$ and the transverse force $F_{y}$ is a function of the spin or the ratio of the normal component of the angular speed of rotation $\omega_{n}$ to the forward locomotive velocity $v$ :

$$
\Phi=\frac{\omega_{n}}{v} .
$$

The appearance of this function is similar to the previous function, but is nevertheless less familiar at an experimental level.

The theoretical adhesion coefficient $\psi$ for Diesel locomotives is calculated by the formula:

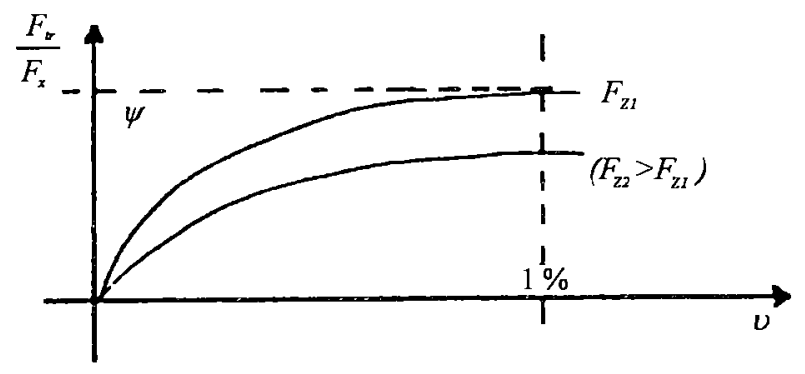

Fig 1. Dependence of slipping $v$ on normal force $F_{z}$

$$
\Psi=0.25+\frac{8}{100+20 v} ;
$$

where $\nu$-velocity of locomotive, $\mathrm{km} / \mathrm{h}$.

$$
\text { Ratio } \mu=\frac{F_{t r}}{F_{z}} \text { shows real diminution of traction force }
$$

parison of theoretical adhesion coefficient $\psi$ and real ratio $\mu=\frac{F_{t r}}{F}$ is presented in Fig 2. It is seen that the value of $\psi$ falls more slowly than ratio $\mu$ when the train speed increases up to $160 \mathrm{~km} / \mathrm{h}$.

The possible tangential force $F_{x}=F_{z} \psi$ is compared with real traction force $F_{t r}$ (Fig 2). It is seen that the difference $F_{x}-F_{t r}$ grows faster when the speed is greater than $50 \mathrm{~km} / \mathrm{h}$. It is the reserve to increase the traction force $F_{t r}$ to get over the aerodynamic resistance $F_{a \dot{r}}$, which rises up rapidly when velocity of vehicle exceeds $150 \mathrm{~km} / \mathrm{h}$. (Fig 3). The aerodynamic resistance is calculated by the formula:

$$
F_{\text {air }}=0.5 c_{x} \rho A v^{2}
$$

where $c_{x}-$,,stream line" coefficient; $\rho$-density of air, $\mathrm{kg} /$ $\mathrm{m}^{3} ; A$ - front area of locomotive, $\mathrm{m}^{2} ; v$-speed of train, $\mathrm{m} / \mathrm{s}$.

\section{Railway dynamics}

\subsection{Principles}

The first application of contact theory concerns what is conventionally referred to as railway dynamics, i.e. the analysis of low-frequency movements of vehicles in terms of both safety and comfort.

As a first approximation a vehicle consists of a set of rigid bodies connected by damped elastic links: axles, bogies and vehicle bodies [6]. Overall movement is therefore regulated by a system of second-order differential equations stated in matrix form:

$$
\{F\}=\{M\}\{\ddot{x}\}+\{K\}\{x\}+\{C\}\{\dot{x}\} ;
$$

where $x$ - the generalised co-ordinates of the system (or degrees of freedom); $M$ - the mass; $K$ - the stiffness; $C$ the viscance; $\{F\}$ - the system of forces applied to the vehicle.

For transverse dynamics, these forces act on the properties of the wheel/rail contact in contrast to vertical dynamics, which only depends on flaws in the track [7]. The roots of the system of equations are of the form:

$$
x=x_{0} e^{p t}, p=\alpha \pm j \omega
$$

where $p$-the root, generally complex, of the characteristic equation of which the degree equals twice the number of degrees of freedom.

If the real part $\alpha$ is negative, the system is stable, i.e. it returns to its position of equilibrium after a disturbance by making a certain number of oscillations that depend on the rate of damping.

In contrast, if the real part is positive, the system is unstable, i.e. it deviates indefinitely from its position of equilibrium following a disturbance. In reality the amplitude of movement is limited by non-linearity in the system (if it were not for the presence of wheel flanges that limit the play of axles in the track). 


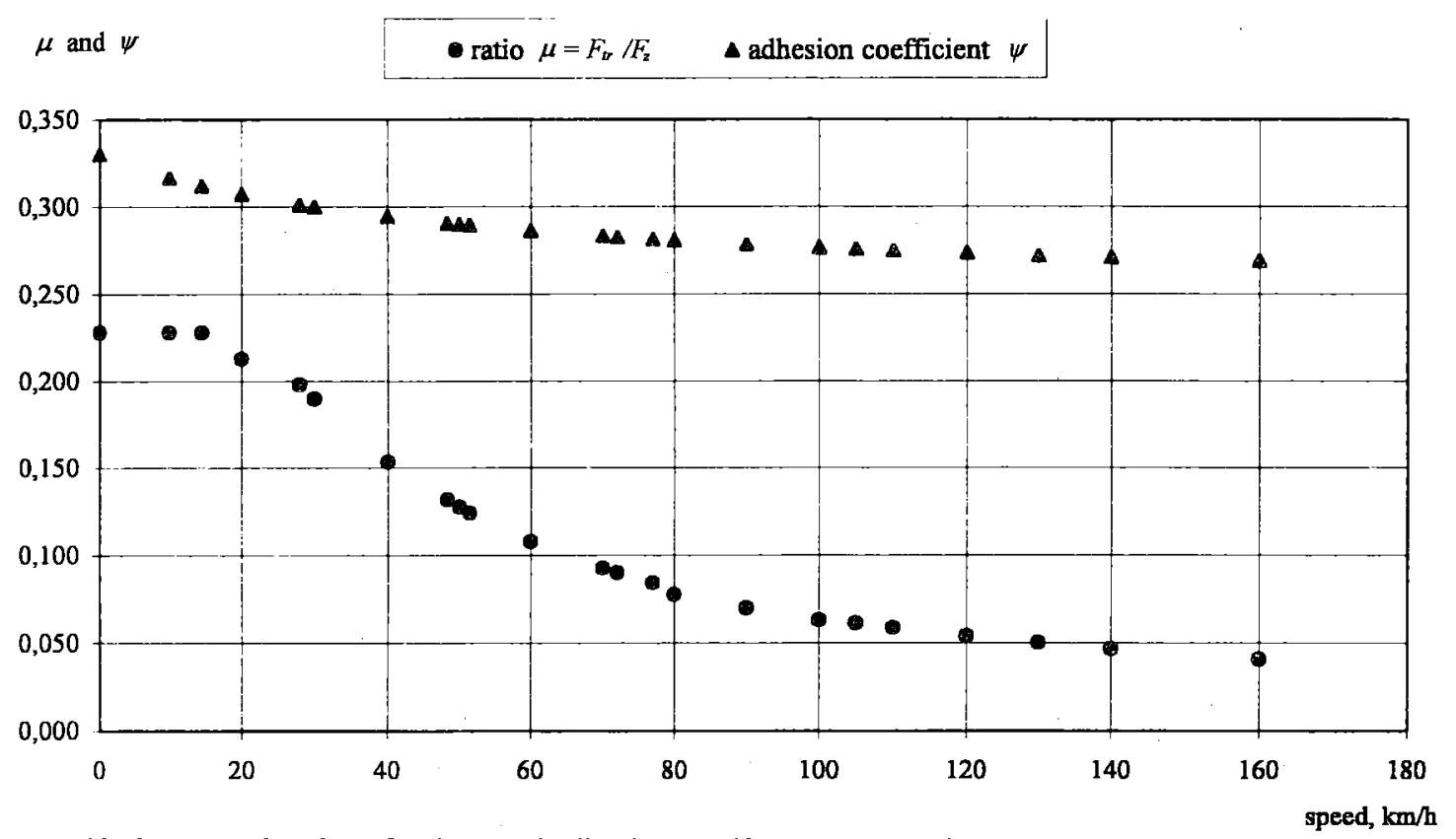

Fig 2. Dependencies of ratio $\mu$ and adhesion coefficient $\psi$ on train speed (locomotive TEP 70)

$F_{x}, F_{i r}, F_{a r}$

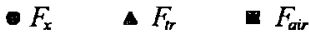

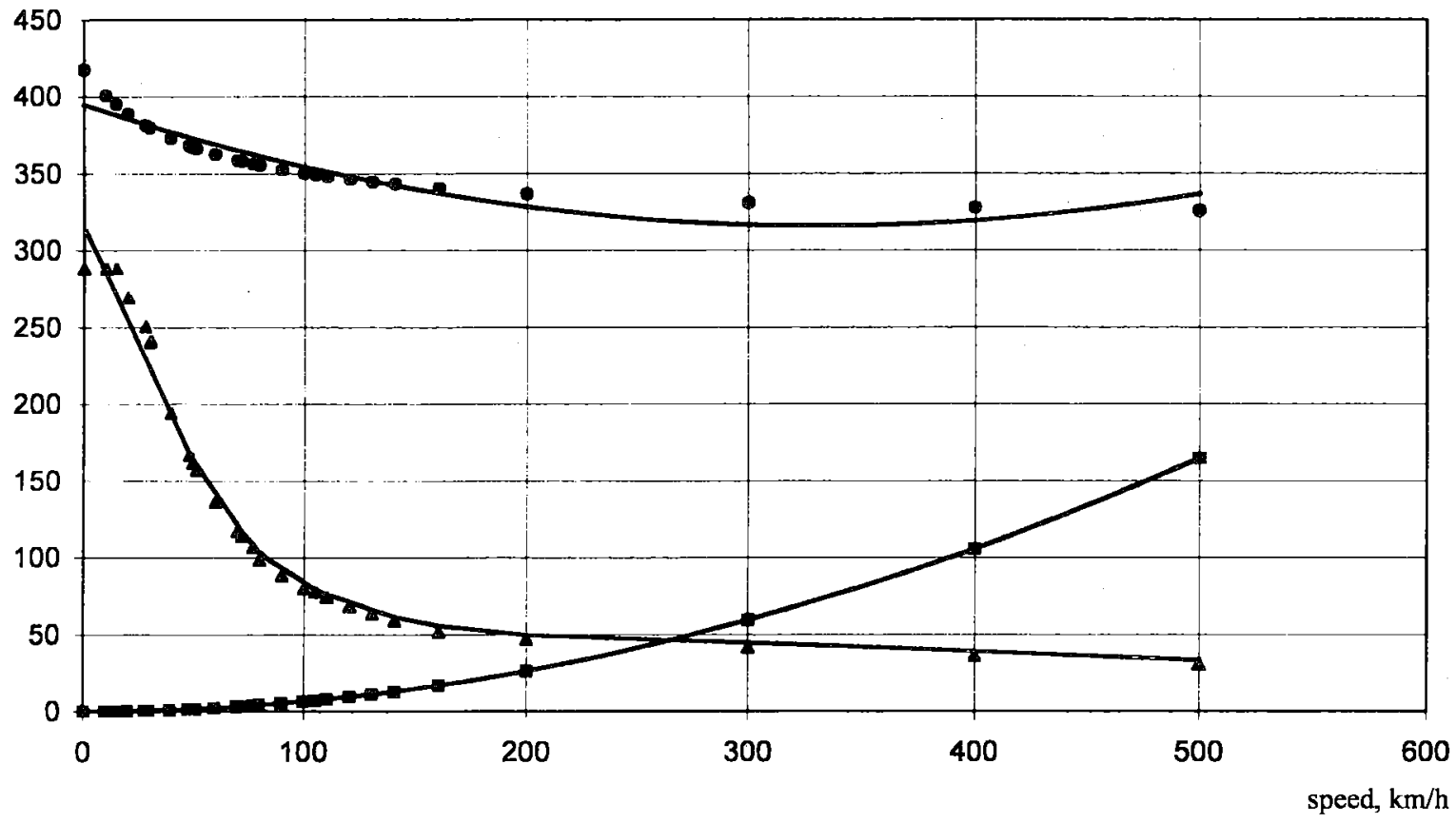

Fig 3. Theoretical tendencies of growing of aerodynamic resistance and decreasing of traction force (locomotive TEP 70)

The forces and accelerations corresponding to these movements affect the subsystems differently depending on their frequency. Generally speaking, one can state that accelerations in the vehicle body determine the comfort of passengers whereas the forces exerted by the axles on the track determine safety. Here only two phenomena that must be avoided as far as safety is concerned are specified: deformation of the track (shifting of the track) under the effect of the total force exerted by the axle [5]. Its limit is stated by formula (8); the notation is the same as in Fig 4:

$\Sigma F_{y}=0.85\left(10+\frac{F_{z}}{3}\right) ;$ 


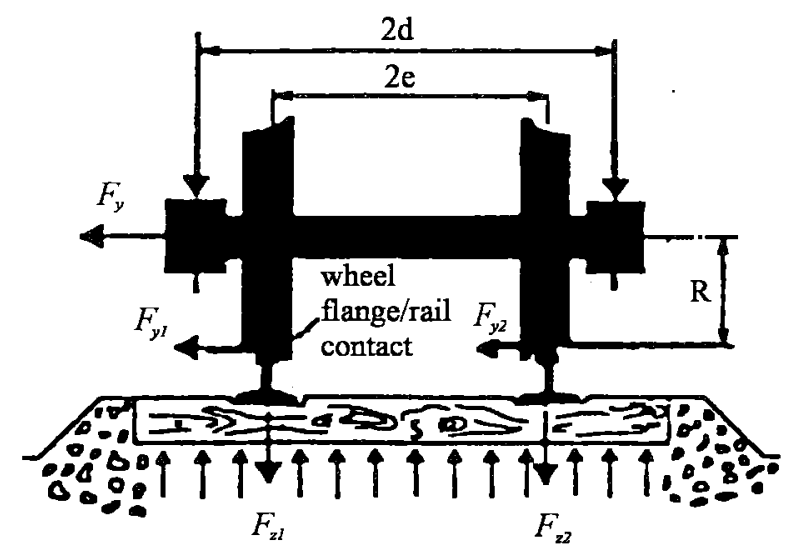

Fig 4. Total forces exerted by wheel-set in contact with the rail

$$
F_{z}=F_{z 1}+F_{z 2}
$$

- derailment due to the wheel flange rising onto the rail; this only depends on the ratio of the (transverse and vertical) forces exerted by the wheel whose wheel flange is in contact with the rail (guiding wheel).

The purpose here is obviously not to describe the complete physical model but simply to show that the origin of instability is located at the level of wheel/rail contact. Analysis of the axle subsystem is sufficient to explain the phenomenon without making claim to precise (or even approximate) numeric results. Thus only running in alignment (or on a curve having a large radius) is considered here, since this makes it possible to assume that the properties of wheel/rail contact are linear.

Arranging the system as an equation requires knowledge of:

- the relationship between forces and slip velocities;

- the slip velocities as a function of the position of points of contact (geometrical analysis).

\subsection{Relationship between forces and slip velocity}

Determination of the relationship between forces, moments and reduced slip, has been carried out by several researchers $[1,8]$ and can be summarised as follows.

The Hertz contact ellipse mentioned earlier in connection with statistical analysis is modified, if there is any relative displacement. It can be divided into two parts (Fig 5):

- a slip area located to the rear where all the points slip in relation to the rail and therefore transmit a limited force;

- an adhesion area in which slip is zero and where the

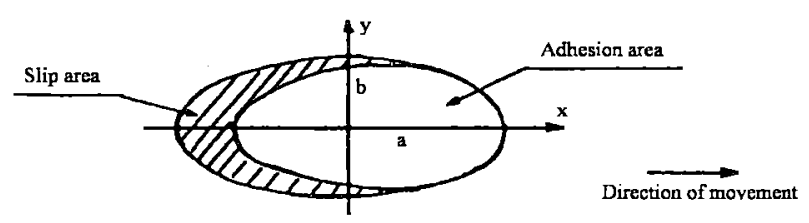

Fig 5. Location of the slip area in wheel/rail contact forces transmitted by each point are less than this limit.

This asymmetry with respect to the transverse axis means that the resultant of the transverse forces $F_{y}$ does not pass through the centre of the ellipse and that there is an additional (resistance motion) moment compared with the moment due to pivoting.

Similarly, pivoting creates unbalanced distribution of contact forces which geometrical sum is not zero. By integrating the tangential stresses over the contact area, Prof. Kalker [5] established the relationship between forces, moments and slip in the range of low slip (less than 1\%):

$$
\left\{\begin{array}{l}
F_{x}=-G a b C_{11} v_{x} ; \\
F_{y}=-G a b C_{22} v_{y}-C_{23} G(a b)^{3 / 2} \phi ; \\
M_{o z}=C_{23} G(a b)^{3 / 2} v_{y}-C_{33} G(a b)^{2} \phi ;
\end{array}\right.
$$

where $a, b$ - the semi axes of the Hertz contact ellipse, $G$ the transverse modulus of elasticity: $G=E / 2(1+\sigma)$, for steel: $E \approx 210000 \mathrm{MPa}$ and $\sigma=0.25$; hence $G \approx 84000$ $\mathrm{MPa} ; C_{11}, C_{22}, C_{23}, C_{33}$ - coefficients calculated by Prof. Kalker that do not depend on the ratio $a / b$.

For a simplified analysis, the following relationships are acceptable:

$$
\left\{\begin{array}{l}
F_{x}=-G a b C_{11} v_{x} \\
F_{y}=-G a b C_{22} v_{y} .
\end{array}\right.
$$

Note that the influence of the vertical load $F_{z}$ is implicitly contained in the product $a b$, which is proportional to $F_{z}^{2 / 3}$.

\subsection{Calculation of slip velocities}

The axle has two degrees of freedom:

- transverse displacement $y$;

- rotation around its centre of gravity in the horizontal plane $\varphi$.

As a first approximation and limiting oneself to the first order:

- rotation $\varphi$ has no effect;

- the transverse displacement $y$ causes displacement of the points of contact that is reflected in a variation of the running radii that, with $r_{0}$, in a centred position, become $r_{1}\left(>r_{0}\right)$ on the right and $r_{2}\left(<r_{0}\right)-$ on the left (Fig 6).

For a conical profile, the variation in the radius is proportional to $y$ :

$$
r_{1}-r_{2}=2 \gamma y ;
$$

where $\gamma$-the angle of the vertex of the cone.

For any profile an equivalent conicity $\gamma^{\prime}$ is defined by linearising the relationship $\Delta r=f(y)$ measured pointby-point over the track and on the axle by means of special rules. 


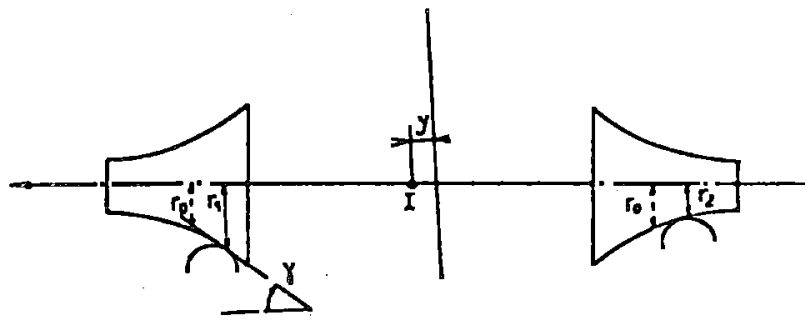

Fig 6. Position of axle in rail track

The slip velocity of the wheel relative to the (stationary) rail is the absolute velocity of the point which coincides with the point of contact. This equals the sum of the drive velocity (translational motion at velocity $v$ and rotation at angular velocity around the centre of gravity) and the relative velocity (angular velocity $\omega=v / r_{0}$ ).

By limiting oneself to the first order, after division by speed $v$ to obtain reduced slip, we have in Fig 6:

on the right: $\left\{\begin{array}{l}v x_{1}=\left(1-\frac{r_{1}}{r_{0}}\right)-\frac{e}{v} \varphi ; \\ v y_{1}=\frac{y}{v}-\varphi .\end{array}\right.$

- on the left: $\left\{\begin{array}{l}v x_{2}=\left(1-\frac{r_{2}}{r_{0}}\right)+\frac{e}{v} \varphi ; \\ v y_{2}=v y_{1} .\end{array}\right.$

where $e-$ a half of gauge, $\mathrm{m}$.

\subsection{Movement of an axle without inertia}

The forces and moments are proportional to the slip velocities and are zero. The differential equation for the movement is:

$$
\ddot{y}+\frac{v^{2}}{2 e r_{0}}\left(r_{1}-r_{2}\right)=0
$$

or if $\Delta r=2 \gamma^{\prime} y$;

then $\ddot{y}+\omega^{2} y=0 \quad \omega^{2}=v^{2} \frac{\gamma^{\prime}}{2 e r_{0}}$;

where $\gamma^{\prime}$-conicity of wheel running surface.

The axle describes a sinusoidal movement along the track having a wave-length:

$$
\lambda=\nu \frac{2 \pi}{\omega}=2 \pi \sqrt{\frac{r_{0} e}{\gamma^{\prime}}}
$$

where $e-$ a half of gauge, $\mathrm{m}$.

This wave-length is a characteristic of the axle. In fact the formula must be modified slightly, if the axle is mounted in a bogie, which gives:

$$
\lambda=2 \pi \sqrt{\frac{r_{0} e}{\gamma^{\prime}}\left(1+\frac{a^{2}}{e^{2}}\right)} ;
$$

where $a-a$ half of the wheelbase, $m ; r_{0}-a$ half of running radius, $m$.

For a locomotive TEP 70 (Russia) axle:

$r_{0}=0.61 \mathrm{~m} ; e \cong 0.76 \mathrm{~m}$ and $a=2.34 \mathrm{~m}$.

For a new wheel tyre profile:

if $\gamma^{\prime}=0.025 \rightarrow \lambda=87.6 \mathrm{~m}$;

if $\gamma^{\prime}=0.05 \rightarrow \lambda=61.9 \mathrm{~m}$.

For a worn wheel tyre profile:

$$
\gamma^{\prime} \cong 0.1 \rightarrow \lambda=43.8 \mathrm{~m} \text {. }
$$

\subsection{Movement of an axle with inertia}

It is assumed that the axle is connected by elastic links having stiffness $K_{x}$ and $K_{y}$ to a roof of infinite inertia moved by a translational motion parallel to the track of velocity $\nu$.

$$
\begin{aligned}
& m \ddot{y}=-2 G a b C_{11} \times\left(\frac{\dot{y}}{v}-\varphi\right)-2 K_{y} \\
& I \dot{\varphi}=-2 e G a b C_{22} \times\left(\frac{e}{v} \varphi+\frac{\gamma}{r_{0}} y\right)-2 K_{x} b^{2} \varphi
\end{aligned}
$$

where $I$-inertia around axle OZ; or, in symbolic notation and rearranging the terms:

$$
\left[p^{2}+\frac{2 G a b C_{11}}{m v}+\frac{2 K y}{m}\right] y-\frac{2 G a b C_{11}}{m} \varphi=0
$$

$$
2 G a b C_{22} \frac{\gamma e}{r_{0}} y+\left[p^{2}+\frac{2 e^{2} G a b C_{22}}{I v} p+\frac{2 K_{x} b^{2}}{I}\right] \varphi=0 .
$$

Making the determinant of the coefficients equal to 0 , gives the characteristic equation of the form:

$$
p^{4}+a p^{3}+b p^{2}+c p+d=0 .
$$

The critical condition is equivalent to the cancelling of one of the real parts of the conjugate square roots:

$$
\alpha_{1} \pm j \omega_{1} \text { and } \alpha_{2} \pm j \omega_{2}
$$

Since the only variable parameter is velocity, this solution therefore corresponds to a critical velocity which value depends on the geometrical and mechanical characteristics of the axle, the elasticity of contact; the stiffness of the links and the equivalent conicity. The literal expression is irrelevant in this case.

\subsection{Wheel/rail interface}

Once all the structural parameters of the vehicle - 
including the axles - have been selected, the only variable capable of modifying the critical velocity is the "equivalent conicity" defined above. Calculations and experience show that the critical velocity depends largely on this quantity. The critical velocity decreases as the conicity increases, all other things being equal.

Construction Division of French National Railways (SNCF) opted for a whee1/rail parameter system providing a low conicity ( 0.025 in the new state) that gives preference to high speeds.

In contrast, Federal German Railway (DB) opted for a relatively high conicity $(0.2)$ that remains constant in time. This choice which at first sight is less favourable for high speed does, on the other hand, have an advantage for wagons having two axles, because they have less lateral stability on the track. Nevertheless, the current tendency is to reduce this value.

All the same, tests carried out in France and Germany have demonstrated that up to $300 \mathrm{~km} / \mathrm{h}$, compatibility of the two systems was achieved for high-speed vehicles on both networks.

The effects of the various parameters of the wheel/ rail interface on the equivalent conicity are given below $[3,5]$ :

- rail profile: standard profile UIC ${ }^{1} 60$ which will be used in future on all heavy traffic or high-speed routes in West Europe is shown in Fig 7.

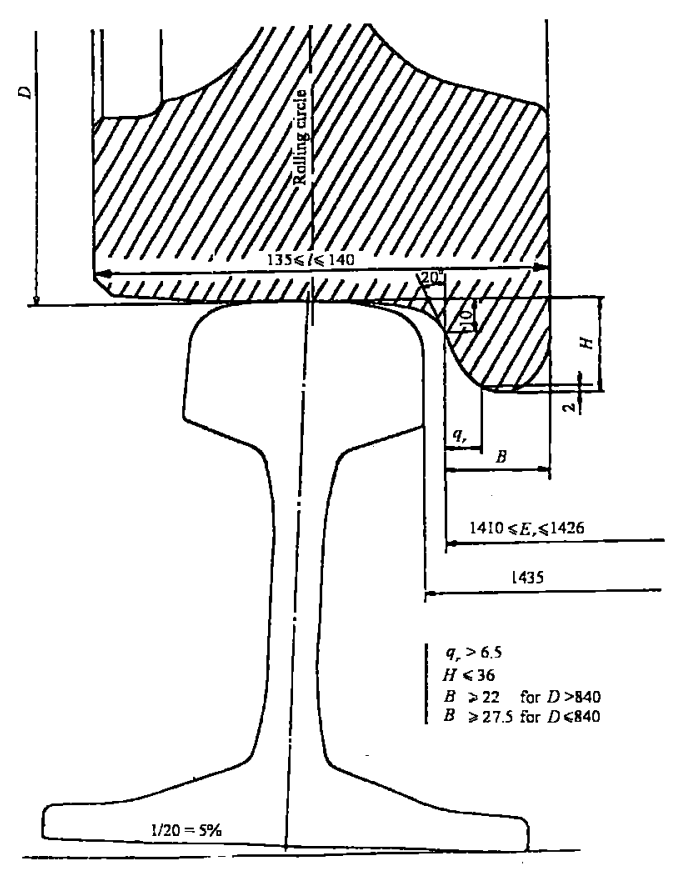

Fig 7. Rail and wheel profile used in West Europe railways

\footnotetext{
${ }^{1}$ UIC - International Union of Railways, formed 1922 to standardise and improve railway equipment and operating methods, with special regards to international traffic.
}

Making allowance for the manufacturing process by rolling and flattening without machining, tolerances in the as-new condition are fairly wide. However, the effect of these tolerances on conicity is not very important;

- inclination of rail relative to vertical is the fundamental parameter. SNCF selected an inclination of $1 / 20$ which moves the point of contact on the wheel from the area where the wheel flange is connected and thus results in low conicity. DB chose an inclination of $1 / 40$ which moves the point of contact closer, thus explaining the higher conicity;

- wheel profile: a standard West European wheel profile (Fig 7) has the distinctive feature of wearing out without deformation (i.e. it has a constant equivalent conicity) when it runs on a track laid with a $1 / 40$ inclination. SNCF decided it was preferable to adopt a conical tread section for the $\mathrm{TGV}^{2}$ with a slope of $\mathrm{l} /$ 40 (hence the conicity of 0.025 in the new state). This profile deforms as it wears out but does so extremely slowly as proved by 10 years of experience with the TGV;

- clearance in the track: this clearance results from the difference between the rail gauge (nominal value 1435 $\mathrm{mm}$ ) and the spacing of the active surfaces of the wheel flanges (maximum value: $1425 \mathrm{~mm}$, minimum value: $1410 \mathrm{~mm}$ ). A clearance of 10 to $15 \mathrm{~mm}$ is considered to be optimal. Less clearance results in excessively high conicity. Greater clearance allows transverse movements of excessive amplitude which, although not hazardous, can be uncomfortable.

\section{Adhesion during traction}

The well-known advantage of steel running on steel is its low resistance to forward motion due to the reduced size of the contact ellipse. In contrast, the forces transmitted between the wheel and rail are limited by the coefficient of adhesion $\psi=\frac{F_{t r}}{F_{z}}$ which, as was seen earlier, depends on numerous factors that cannot always be controlled. Important study work was carried out by $\mathrm{ORE}^{3}$ Committee and several essential details from it are presented here.

The force that can be transmitted for a given load increases with reduced slip in accordance with the characteristics shown earlier in chapter 2.2., but beyond a certain value $v_{n}$ (which is referred to as optimum slip), it decreases sharply and skidding occurs.

The maximum value $\psi$ of $F_{t r} / F_{z}$ and the correspond-

\footnotetext{
2 TGV - high speed passengers electric trains and train service in French.

${ }^{3}$ ORE - Research and Trials Office. A UIC organisation which pools the means and results of research and trials carried out by member railway administrations.
} 
ing slip $v_{o}$ depends, to varying extents, on:

- surface condition;

- $\quad$ surface pollution (chemical, dead leaves, etc.);

- atmospheric conditions (temperature, humidity, etc.);

- vertical load $F_{z}$

- vehicle speed $v$.

This last relationship has been the subject of numerous tests which show a reduction in $\psi$ and an increase in slip $v_{0}$ when the speed $v$ increases.

Among the methods hitherto devised in order to increase adhesion, only sanding has proved to be convenient and efficient and remains in general use. In contrast the introduction of electronics and microcomputers has allowed significant progress in the fight against skidding thanks to extremely sensitive detection and rapid response matched to the value of the traction force.

Tests at speeds exceeding $500 \mathrm{~km} / \mathrm{h}$ have given adequate proof [5], that at high train speed is still sufficient to allow the necessary traction forces. The ratio $F_{t r} / F_{z}$ has reached 0.07 without any skidding, thus proving that the limit was not reached.

\section{Conclusions}

1. It should be emphasised that adhesion at train high speed is still sufficient to allow the necessary traction forces because during arising velocity the adhesion coefficient $\psi$ falls more slowly than ratio

$$
\mu=\frac{F_{t r}}{F_{r}} \text { (Fig 2). }
$$

2. The critical velocity of train increases as the wheel conicity is diminished, all other things being equal.

3. Current tendency is to reduce the conicity of wheels with the aim of increasing speed of trains.

4. Inclination of rail relative to vertical is the funda- mental parameter in wheel/rail contact system.

5. Clearance in the track of 10 to $15 \mathrm{~mm}$ is considered to be optimal.

6. Wave-length of moving wheel-set in track for worn wheel tyre is twice less than for new wheel running profile. Hence the rail and wheel flange contact occurs more frequently.

7. Further research on wheel running surface wearing intensity dependence on parameters of wheel-sets sinusoidal movement is indispensable.

\section{References}

1. Szolc T. Simulation of Dynamic Interaction between the Railway Bogie and the Track in the Medium Frequency Range. Multibody System Dynamics, No 6, Kluwer Academic Publishers, 2001, p. $99-122$.

2. Krylov V. V. Ground vibration boom from high-speed trains. Journal of Low Frequency Noise, Vibration and Active Control (United Kingdom), No 18 (4), 1999, p. $207-218$.

3. Zhai W. M., Cai C. B., Wang Q. C. , Lu Z. W., Wu X. S. Dynamic effects of vehicle on tracks in the case of raising train speeds. Journal of Rail and Rapid Transit, Vol 215. IMechE, 2001, p. 125 - 135.

4. Anderson C., Dahlberg T. Wheel/rail impacts at a railway turnout crossing. Journal of Rail and Rapid Transit, Vol 212. IMechE, 1998. p. $123-134$.

5. Moreau A. Characteristics of wheel/rail contact. Rail Engineering. International Edition, 1992, No 3, p. 15-22.

6. Williams D. Prediction of damping in railway wheels with constrained viscoelastic layers. Internal Report, British Rail Research, 1995, 120 p.

7. Frohling R. D., Scheffel H., Ebersohn W. The vertical dynamic response of a rail vehicle caused by track stiffness variations along the track. Vehicle System Dynamics, Vol 25, 1996, p. $175-187$.

8. Knothe K., Grassie S. L. Modelling of railway track and vehicle/track interaction at high frequencies. Vehicle System Dynamics, Vol 22, 1993, p. $209-262$. 